\title{
Higgs searches at the LHC
}

\author{
Koji Nakamura for the CMS and ATLAS Collaboration \\ ICEPP, University of Tokyo, Hongo 7-3-1 Bunkyo-ku, 113-8654 Tokyo, Japan \\ DOI: http://dx.doi.org/10.5689/UA-PROC-2010-09/55
}

\begin{abstract}
During the operation at the center-of-mass energy of $7 \mathrm{TeV}$, the LHC is expected to deliver up to $1 \mathrm{fb}^{-1}$ of $p p$ collision data by the end of 2011. Prospects for the Higgs searches at the LHC with $1 \mathrm{fb}^{-1}$ of integrated luminosity are discussed. The most promising results are expected in two Higgs boson search channels: Standard Model Higgs boson decay into two $W$ bosons and MSSM Higgs decay into two $\tau$ leptons.
\end{abstract}

\section{Introduction}

The Higgs mechanism is a proposed solution to the electroweak symmetry breaking mystery which predicts the existence of an undiscovered particle (Higgs boson, $\mathrm{H}$ ) in the Standard Model (SM) or five such $\left(h, H, A, H^{+}\right.$and $\left.H^{-}\right)$in the Minimal Supersymmetric Standard Model (MSSM). Its discovery is the primary physics goal of the Tevatron and LHC physics program and the discovery potential has been studied extensively for the LHC running at its design center-of-mass energy of $\sqrt{s}=14 \mathrm{TeV}$.

The excellent start-up of the LHC in 2010 at $\sqrt{s}=7 \mathrm{TeV}$ allowed for accumulating $4 \mathrm{pb}^{-1}$ of data at the time of this conference time ${ }^{1}$ About $1 \mathrm{fb}^{-1}$ of integrated luminosity is expected by the end of 2011. The first part of this report describes the reach of the CMS and ATLAS Higgs boson searches for this energy/luminosity scenario. The second part describes the measurement of the lepton identification performance using real data, which is the first step towards the background estimation for the Higgs boson searches.

\section{Sensitivity of the Higgs searches}

The CMS and ATLAS sensitivity results based on Monte Carlo simulation are obtained by scaling the full, detailed $10 \mathrm{TeV}$ (or $14 \mathrm{TeV}$ ) analyses $[1,2,3,4]$ to $7 \mathrm{TeV}$ using cross-section ratio at the two centre-of-mass energies, having checked that the event selection efficiencies are stable between the two energies. The resulting CMS and ATLAS sensitivity to the Higgs boson searches are expressed in terms of expected upper limits at a $95 \%$ confidence level, in selected representative channels [5, 6]. These are Standard Model channels $H \rightarrow W W, H \rightarrow Z Z \rightarrow 4 \ell$, and $H \rightarrow \gamma \gamma$ and the MSSM channel $H / A \rightarrow \tau \tau$. Since the most sensitive Standard Model channel is the the $H \rightarrow W W$ decay, we first show the results for this individual channel, before combining with the other two SM channels. In addition, we present the results for the MSSM Higgs boson search in the most promising $H / A \rightarrow \tau \tau$ decay channel.

\footnotetext{
${ }^{1}$ Up to now (Nov 2010), the LHC accumulated $45 \mathrm{pb}^{-1}$ of data
} 


\section{$2.1 \quad H \rightarrow W W \rightarrow l \nu l \nu$}

\begin{tabular}{ll}
\hline \hline SM $W W$ & 55.2 \\
top & 14.0 \\
$W+$ jets & 5.6 \\
\hline Total background & 74.8 \\
Signal $\left(M_{H}=160 \mathrm{GeV}\right)$ & 39.5 \\
\hline \hline
\end{tabular}

Table 1: Estimated number of events for the $H \rightarrow W W$ signal and the major backgrounds at an integrated luminosity of $1 \mathrm{fb}^{-1}$ after full event selection.
We considered two methods for the extrapolation of the $H \rightarrow W W$ results from 10 to $7 \mathrm{TeV}$ : by scaling according to the cross-section ratios or PDF re-weighting $[5,6]$. The cross-section scaling is motivated by earlier studies showing that the selection efficiencies for the Higgs signal $g g \rightarrow H \rightarrow W W$ and the SM $W W$ background are effectively constant for $\sqrt{s} \geq 6 \mathrm{TeV}$. The PDF re-weighting method, which is used here as a cross check of the cross-section scaling, is performed and results are very similar.

Expected number of SM $H \rightarrow W W$ events

with a Higgs bosom mass of $160 \mathrm{GeV}$ and the corresponding major backgrounds at an integrated luminosity of $1 \mathrm{fb}^{-1}$ after full event selection are shown in Table 1 . The expected $95 \%$ CL upper limits on the product $\sigma_{H} \times \operatorname{Br}(H \rightarrow W W)$ of the Higgs boson production cross-section and its decay branching ratio normalized to the Standard Model prediction are given in Figure 1. The left-hand figure shows the result obtained by the CMS experiment, while the right-hand figure is the result by the ATLAS experiment at different integrated luminosity scenarios at $\sqrt{s}=7 \mathrm{TeV}$.
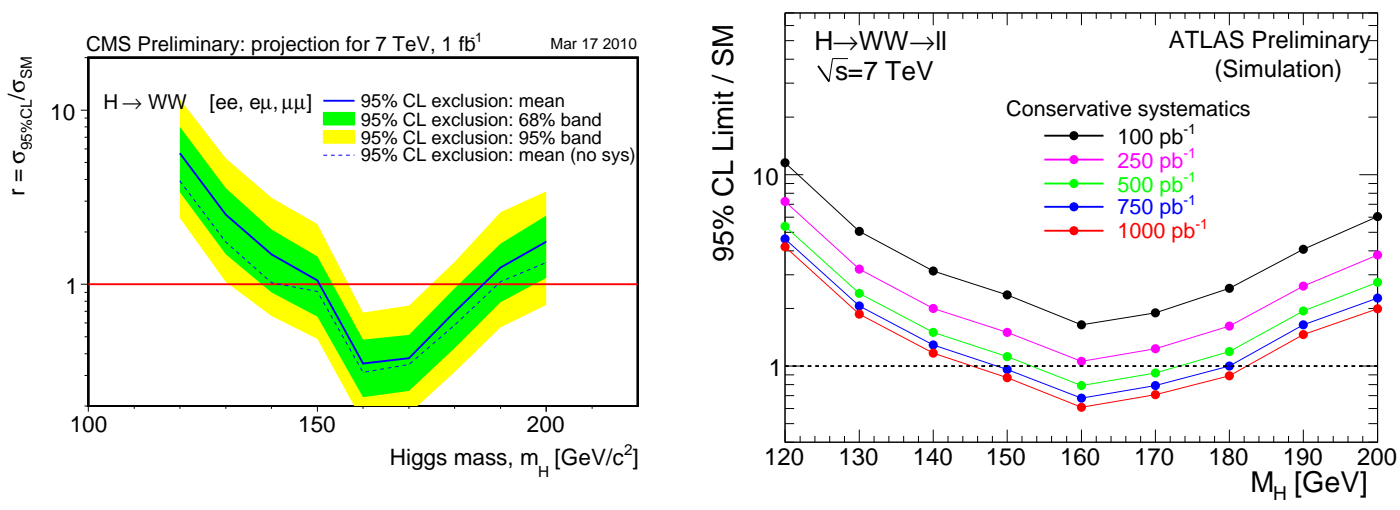

Figure 1: Expected 95\% CL upper limits on the Higgs boson production rate normalized to the Standard Model prediction.

\subsection{Combination of the SM $H \rightarrow W W, H \rightarrow Z Z$ and $H \rightarrow \gamma \gamma$ channels}

Experimentally, the cleanest signature for the SM Higgs boson search is its "golden" decay into four leptons: $H \rightarrow Z Z \rightarrow 4 \ell$, with $\ell=e, \mu$. An excellent energy and momentum resolution for the reconstructed electrons and muons, respectively, leads to a narrow four-lepton invariant mass peak on top of a smooth background. The major component of the background is the irreducible $Z Z \rightarrow 4 \ell$ process. The highest sensitivity in this channel is obtained for Higgs boson 
mass of about $200 \mathrm{GeV}$ where an upper bound in the cross section times the branching ratio of 2 and $2.5 \times\left(\sigma_{H} \times \operatorname{Br}(H \rightarrow 4 \ell)\right)$ is expected by CMS and ATLAS, respectively. The difference is due to the different signal cross section used : the NNLO calculation is used by the CMS experiment while ATLAS relies on the NLO calculation here.

Additional decay channel which can help to improve the sensitivity in the low-mass Higgs region is $H \rightarrow \gamma \gamma$ decay. The $H \rightarrow \gamma \gamma$ analysis strategy for the early data is based on an inclusive approach analysis with the reconstructed di-photon mass as the sole discriminating variable, for the sake of simplicity and robustness. Studies with fully simulated background samples at $7 \mathrm{TeV}$ are performed. Three main processes contribute to the irreducible background at tree-level: the Born process $q \bar{q} \rightarrow \gamma \gamma$, the bremstrahlung process $q g \rightarrow q \gamma \gamma$, the box process $g g \rightarrow \gamma \gamma$. The reducible backgrounds are inclusive prompt-photon ( $\gamma$-jet) and multi-jet production processes with one or more jet(s) mis-identified as photon(s). The reducible background cross-section is initially several orders of magnitude larger than that of the irreducible background. This analysis therefore crucially relies on the detector's capability to discriminate those reducible backgrounds. An upper bound in the cross section times the branching ratio of 4 and $6 \times\left(\sigma_{H} \times B r(H \rightarrow \gamma \gamma)\right)$ is expected by CMS and ATLAS, respectively. Similarly as above, the observed difference is due to different higher-order signal cross section calculations by the two experiments.

Three SM Higgs boson decay modes are considered in combination: $H \rightarrow W W \rightarrow l \nu l \nu, H \rightarrow$ $Z Z \rightarrow 4 \ell$, and $H \rightarrow \gamma \gamma$. The combined Higgs boson exclusion limits presented here are based on a fitting algorithm which uses one signal normalization parameter for all channels. Figure 2 shows the expected 95\% CL upper bound on the Higgs boson production rate normalized to the Standard Model prediction, as a function of Higgs boson mass. The left-hand figure shows the CMS experiment results and right-hand figure shows the results from ATLAS experiment. At the luminosity and center-of-mass energy used, a Standard Model Higgs boson with a mass region $145-190 \mathrm{GeV}$ and $135-188 \mathrm{GeV}$ can be excluded by CMS and ATLAS, respectively. ${ }^{2}$
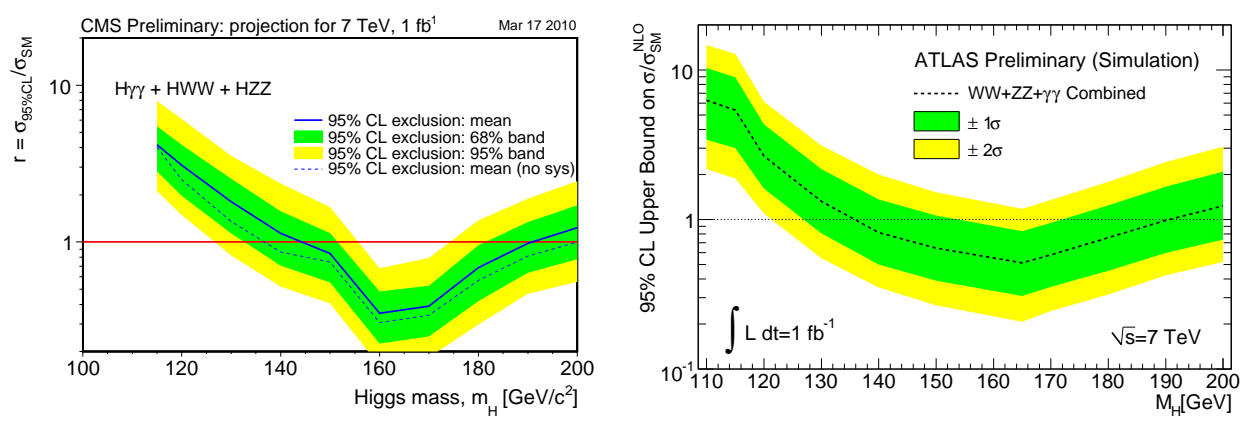

Figure 2: Expected 95\% CL upper limits of the Higgs boson production rate normalized to the Standard Model prediction.

\footnotetext{
${ }^{2}$ At the writing time of this documet, an update result of the ATLAS Higgs sensitivity projections with improvements is available. [7]
} 


\section{$2.3 \quad$ MSSM $H \rightarrow \tau \tau$}

The result of the projected sensitivity for the MSSM Higgs Bosons in the $p p \rightarrow b b \Phi, \Phi \rightarrow \tau \tau$ $(\Phi \equiv h, H, A)$ search is described in this section. The following three final states were used $: \tau_{\mu} \tau_{\text {had }}, \tau_{e} \tau_{\text {had }}, \tau_{\mu} \tau_{e}$, where $\tau_{\mu}$ stands for $\tau \rightarrow \mu \nu \nu$ decay, $\tau_{e}$ stands for $\tau \rightarrow e \nu \nu$, and $\tau_{\text {had }}$ stands for the hadronic $\tau$-decays with one or three charged pions. The analysis strategy is based on the use of b-tagging for the Drell-Yan background suppression, reconstructing the final $\tau \tau$ final states, and counting events in optimal $m_{\tau \tau}$ mass windows. Figure 3 shows the expected sensitivity of such a search interpreted in the context of the MSSM $m_{h}^{\max }$-scenario. At $\sqrt{s}=$ $7 \mathrm{TeV}$ and with a $1 \mathrm{fb}^{-1}$ of data, one can expect to reach a discovery level sensitivity in a large, so far unexplored range of the $\left(m_{A}, \tan \beta\right)$-plane, covering $\tan \beta>20$ at low $m_{A}$. In the absence of signal, the exclusion range covers even a larger area down to the values of $\tan \beta \sim 15$ at low $m_{A}$.

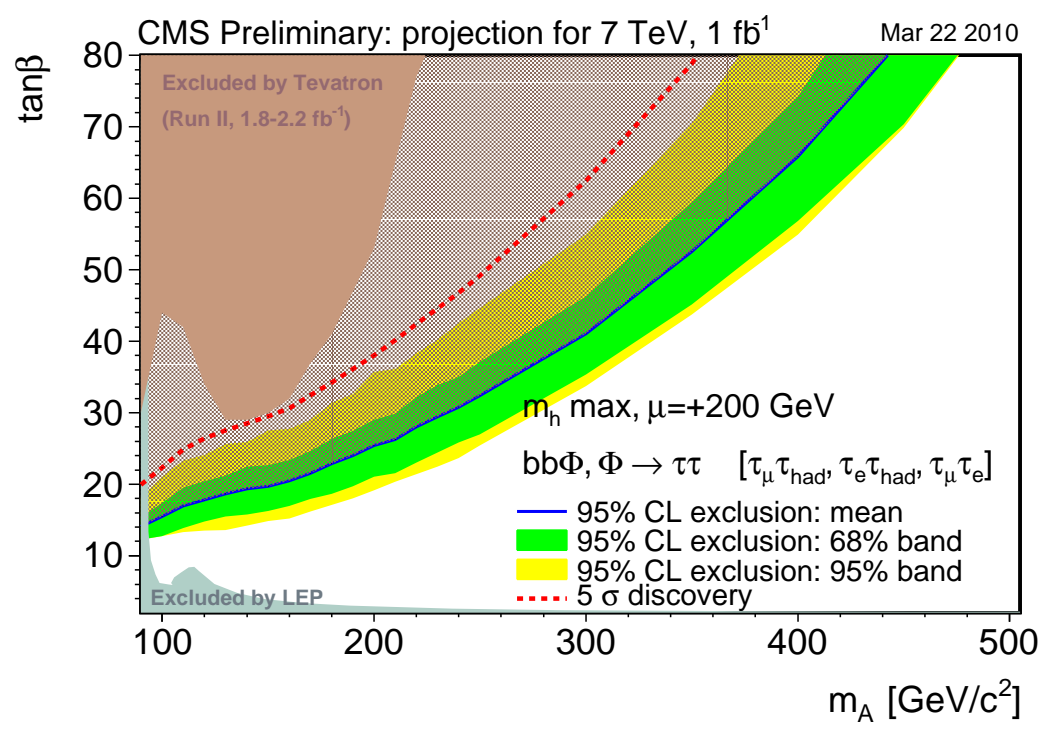

Figure 3: Expected sensitivity to the MSSM Higgs bosons in the $p p \rightarrow b b \Phi, \Phi \rightarrow \tau \tau$ search. The red dotted line indicates the range for a discovery-level (5-sigma) sensitivity. The solid blue line defines the range expected to be excluded at $95 \%$ C.L. in absence of signal. Regions currently excluded by LEP and Tevatron are also shown.

\section{Lepton Performance study with real data}

Using the $4 \mathrm{pb}^{-1}$ of data accumulated by both CMS and ATLAS detectors, one can study the reconstruction performance of the final state particles. Especially for the two of the most promising channels, $H \rightarrow W W$ and MSSM $H / A \rightarrow \tau \tau$, the efficiency and fake rate performance for the final state leptons are measured.

It is useful to consider a definition of the fake lepton rate as $f_{l} \equiv \frac{N_{\text {identified object }}}{N_{\text {loose criteria }}}$, where 

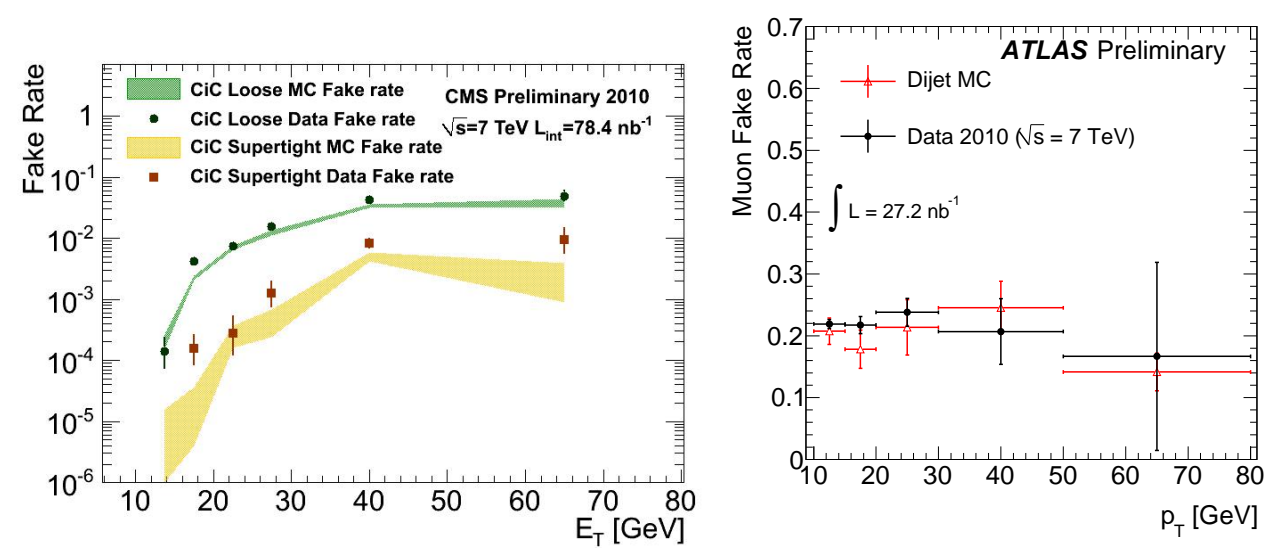

Figure 4: The electron (left) and muon (right) fake rate as a function of the lepton transverse momentum, as measured by the CMS and ATLAS experiment respectively. The fake rate obtained from data is compared with the fake rate from Monte Carlo.

$N_{\text {loose criteria }}$ is the number of objects passing certain loose criteria, while $N_{\text {identified object }}$ is number of objects which in addition pass certain stricter identification criteria. A dijet sample is one suitable data set for the calculation of the fake lepton rate due to the relatively small contamination by real leptons from $W$ and $Z$ bosons decays. The fake electron and muon rates are shown in Figure 4 as a function of the lepton transverse momentum, as measured by the CMS and ATLAS experiments [8][9]. The fake electron rate is calculated for identified electrons with respect to all reconstructed electron candidates. The muon fake rate is defined for isolated muons with respect to all muon candidates without any isolation requirement.

\section{Conclusion}

The estimated CMS and ATLAS sensitivities to Higgs bosons in selected channels at $7 \mathrm{TeV}$ and $1 \mathrm{fb}^{-1}$ are presented. The combined analysis of Standard Model channels, $H \rightarrow W W$, $H \rightarrow Z Z \rightarrow 4 \ell$, and $H \rightarrow \gamma \gamma$ should allow for an exclusion of a Standard Model Higgs boson at a $95 \%$ confidence level in a Higgs mass range of $145-190 \mathrm{GeV}(135-188 \mathrm{GeV})$ with CMS (ATLAS) experiments, respectively. The sensitivity in this mass range is largely dominated by the $H \rightarrow W W$ channel. For the MSSM Higgs, we can expect to reach the discovery level sensitivity in a large, up to now partly unexplored range of the $\left(m_{A}, \tan \beta\right)$-plane, covering $\tan \beta>20$ at low $m_{A}$, at $7 \mathrm{TeV}$ and with a $1 \mathrm{fb}^{-1}$ of integrated luminosity. In the absence of signal, the exclusion range covers an even larger range down to $\tan \beta$ values of about 15 at low $m_{A}$.

The lepton performance studies with real data are ongoing and the lepton fake rate measurement is presented as an example. These studies provide a starting point for upcoming Higgs boson searches based on real data. 


\section{References}

[1] CMS Collaboration, CMS Technical Design Report, Vol. II: Physics Performance, J. Phys. G 34, 995 (2007).

[2] CMS Collaboration, http://cdsweb.cern.ch/collection/CMS Physics Analysis Summaries?ln=en

[3] ATLAS Collaboration, Expected Performance of the ATLAS Experiment - Detector, Trigger and Physics, arXiv:0901.0512 [hep-ex].

[4] ATLAS Collaboration, Prospects for Higgs Boson Searches using the $H \rightarrow W W(*) \rightarrow l \nu l \nu$ Decay Mode with the ATLAS Detector for $10 \mathrm{TeV}$, ATL-PHYS-PUB-2010-005.

[5] CMS Collaboration, The CMS physics reach for searches at 7 TeV, CERN-CMS-NOTE-2010-008.

[6] ATLAS Collaboration, ATLAS Sensitivity Prospect for Higgs Boson Production at the LHC Running at $7 \mathrm{TeV}$, ATL-PHYS-PUB-2010-009.

[7] ATLAS Collaboration, ATLAS Sensitivity Prospects for 1 Higgs Boson Production at the LHC Running at 7, 8 or $9 \mathrm{TeV}$, ATL-PHYS-PUB-2010-015.

[8] CMS Collaboration, Electron Reconstruction and Identification at $\sqrt{s}=7 \mathrm{TeV}$, CMS-PAS-EGM-10-004.

[9] ATLAS Collaboration, Observation of the Background from $W+$ jets to Search $H \rightarrow W W \rightarrow \ell \nu \ell \nu$ with the ATLAS detector at $7 \mathrm{TeV}$, ATLAS-CONF-2010-092. 